\title{
New therapy for chemotherapy-induced hepatic failure in leukemia; a randomized double-blind clinical trial study
}

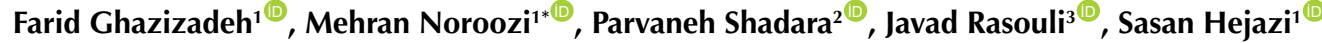 \\ 'Department of Pediatric Oncology, University of Medical Sciences of Urmia, West Azerbaijan, Iran \\ ${ }^{2}$ Department of Pediatrics, University of Medical Sciences of Urmia, West Azerbaijan, Iran \\ ${ }^{3}$ Department of Biostatistics, University of Medical Sciences of Urmia, West Azerbaijan, Iran
}

${ }^{*}$ Correspondence to

Mehran Noroozi, Email:

noroozi.m@umsu.ac.ir,

mehranxnoroozi@gmail.com

Received 29 April 2020 Accepted 20 July 2020 Published online 29 Aug. 2020

Keywords: Hepatotoxicity, Chemotherapy, Acute lymphoblastic leukemia, Milk thistle

\begin{abstract}
Introduction: Hepatotoxicity is usual toxicity after chemotherapy in acute lymphoblastic leukemia (ALL) patients. Conventional treatment methods such as supportive care did not have an effective role in the improvement of hepatotoxicity.

Objectives: In this study clinical efficacy of milk thistle in contrast with placebo was compared in leukemia patients with chemotherapy-induced hepatotoxicity.

Patients and Methods: In this double-blind study, 93 ALL patients with chemotherapy-induced hepatotoxicity were randomized to a clinical trial with milk thistle or placebo. Liver enzymes level evaluated during 70 days. We divided patients randomly into two groups. Milk thistle at dosage of $7 \mathrm{mg} / \mathrm{kg}$ daily was prescribed in the intervention group while in the control group, placebo pills similar to milk thistle in shape and color were prescribed daily.

Results: At day 35 and day 70 of the study, in the milk thistle arm ALT and AST mean serum levels were lower than the placebo group $(P<0.001)$. In the milk thistle group there was a significant reduction in mean of AST and ALT during the first 35 days in patients who were taking livergol in comparison to next 35 days that patients stopped taking it. Children's age was between 3-15 years.

Conclusion: Based on our results, milk thistle improves liver function in chemotherapy-induced hepatotoxicity and there was no need for dose reduction or discontinuation of chemotherapy. Future clinical trials should be conducted to explore longtime effect of livergol in leukemia patients and to determine if there is any need for prophylactic administration of antioxidants.

Trial Registration: This clinical trial has been approved by the Iranian Registry of Clinical Trials at 2018-02-14 (identifier: IRCT20170821035831N2; http://en.irct.ir/trial/26957).
\end{abstract}

\section{Introduction}

Acute lymphoblastic leukemia (ALL) is the most common malignancy in children. It accounts for one-fourth of all cancers and 72\% of all cases of childhood leukemia (1). More than $80 \%$ of patients improve with systemic chemotherapy and prophylactic central nervous system chemotherapy. However, systemic chemotherapy of ALL results in a significant morbidity and mortality $(3,4)$, since neurotoxicity, liver dysfunction and mucositis are serious side effects (5-7). Metabolites of 6-mercaptopurine and methotrexate are the main cause of hepatotoxicity in maintenance phase of ALL chemotherapy (8).

Historically, over 2000 years milk thistle has been used for liver diseases such as hepatitis, cirrhosis and gallbladder problems. Some studies suggest that milk thistle is applied as a supportive care agent in conditions with liver and kidney toxicity (9-11).

\begin{abstract}
Key point
Every dose reduction or chemotherapy discontinuation in leukemia treatment course increases the risk of remission failure and leukemia relapse. Liver failure is one of the most common causes of dose reduction. Milk thistle will improve liver function and reduce chemotherapy hepatotoxicity.
\end{abstract}

Anti-inflammatory, anti-apoptotic, and anti-oxidative as well as inhibition of tumor necrosis factor (TNF-a) release and caspase- 3 activation, increase of the antiapoptotic protein $\mathrm{Bcl}-2$ level, proteases, and hyaluronidase effects are some possible mechanisms for milk thistle protective effects (12).

\section{Objectives}

There is not enough prospective study about the efficacy of milk thistle in chemotherapy-

\footnotetext{
Copyright $(\subset 2021$ The Author(s); Published by Nickan Research Institute. This is an open-access article distributed under the terms of the Creative Commons Attribution License (http://creativecommons.org/licenses/by/4.0), which permits unrestricted use, distribution, and reproduction in any medium, provided the original work is properly cited.
} 
induced hepatotoxicity; therefore, in this study we evaluated and compared clinical efficacy of milk thistle in contrast with placebo on chemotherapy-induced hepatotoxicity in children with ALL.

\section{Patients and Methods}

Our study was conducted as a prospective double-blind clinical trial in oncology department from November 2016 to May 2017. Patients' diagnoses made based on clinical findings, review of initial bone marrow aspiration slides and flow cytometry results.

In our department, in maintenance phase, every 35 days patients have an injection of vincristine at day 0 which followed by 5 days steroid pulses and weekly methotrexate (MT) $20 \mathrm{mg} / \mathrm{m}^{2}$ and daily mercaptopurine $75 \mathrm{mg} / \mathrm{m}^{2}$ for two years in girls and three years in boys. As our routine, we check liver enzymes level and bilirubin level before injection of vincristine at day 0 of all courses for all of ALL patients in maintenance phase of chemotherapy. Patients In this study in two arms of intervention and control, treated with MT and placebo for 35 days, respectively, and in additional 35 days the treatment followed. In order to control confounding parameters, we used double blind method, in which all individuals and physicians who prescribed the drugs were not aware of the type of drugs. Additionally, our biostatistics analyzer was not aware of our grouping, only the first author had knowledge of the randomization assignment. The samples were selected by the convenience method among patients of oncology department and allocated in two arms while a block randomization procedure was used with matched subjects in each block based on sex and age. Thus, at first, the size of the blocks was determined to be six in this study. In the next step, according to the sample size, the number of the required blocks was considered to be 15 . In the next step, all the possible combinations were listed and, for each, a code was assigned. Among these codes, 15 codes were selected using the drawing method and listed in order. The assignment of the individuals was based on the combination of the selected blocks, such that in addition to the random nature and lack of prediction on the assignment of the participants to the intervention and control groups, the size of each group remained equal.

All parameters were measured on days 0,35 and 70 during their routine visit for chemotherapy and no additional blood sampling imposed on the patients.

\section{Hepatotoxicity criteria}

The grading system applied to patients, adapted from the American National Cancer Institute grading scale for Hepatotoxicity, published in 1999. Patients with grade II and III entered into the study and patients with hepatotoxicity grade IV were not included in the study.

\section{Milk thistle}

Milk thistle is scientifically called Silybum marianum. It is a plant of the Asteraceae family. Originally a native of Southern Europe and Asia, it is now found throughout the world. Studies suggest that milk thistle prevents toxin entry to hepatocytes, stimulates protein synthesis and accelerates hepatocytes regeneration. In patients with hepatitis $\mathrm{C}$ virus milk thistle showed antiviral effects. Silymarin was safe and well tolerated up to $2.1 \mathrm{~g}$ per day orally. It was safe for up to 41 months use. Gastrointestinal problems ( diarrhea, dyspepsia, nausea, flatulence, anorexia, changes in bowel habits, abdominal bloating, abdominal fullness or pain), headache, skin reactions (pruritus, rash, urticaria, and eczema), neuropsychological events ( asthenia, malaise and insomnia), rhinoconjunctivitis (nasal congestion, runny nose and sneezing), arthralgia (pain in joint), impotence and anaphylaxis (serious life threatening allergic reaction) are rare side effects associated with milk thistle (16-19). In our country milk thistle is sold under the brand of Livergol and was effective in fatty liver and hepatitis with strong antioxidant activities. Livergol has two types of $140 \mathrm{mg}$ and $70 \mathrm{mg}$ coated tablets (each tablet containing $140 \mathrm{mg}$ and $70 \mathrm{mg}$ Silybum marianum, respectively) (20).

\section{Eligibility}

All complications were described to parents and were presented in written form. Follow-up was done using phone contacts and all complications were checked in each visit (physical examination). Any side-effects related to milk thistle monitored, through weekly phone contacts.

\section{Inclusion criteria}

It consisted of pediatric subjects aged between 3-15 years referred to oncology department with a diagnosis of pre-B cell and early pre-B cell ALL ( initial bone marrow involvement with more than $20 \%$ lymphoblasts) in maintenance phase of chemotherapy, with hepatotoxicity grade 2 and 3 according to Common Toxicity Criteria Version 2.0 of National Cancer Institute (NCI) (14).

\section{Exclusion criteria}

It consisted of subjects with obstruction of extrahepatic biliary tract, severe failure of liver or kidney, gastrointestinal obstruction, malabsorption syndrome, grade IV hepatotoxicity, viral induced hepatitis and sepsisinduced hepatotoxicity, denied to participate in study, previous history of allergy to milk thistle, age below 3 years or more than 15 years. We also excluded patients who were no longer willing to stay and collaborate in the study.

\section{Ethical issues}

The research followed the principles of the Declaration of Helsinki. The study was registered in the Iranian Registry of Clinical Trials (identifier: IRCT20170821035831N2; http://en.irct.ir/trial/26957). The ethics committee of the Urmia University of Medical Sciences approved the study (\#IR.UMSU.Rec.1396.5). The process of the study was explained to the patients and informed consent was obtained from all of them.

\section{Data analysis}

Data of patients who completed the trial were analyzed and 
reported. SPSS version 22 software (SPSS Inc., Chicago, IL, USA) was used for Statistical analysis of data. Chi-square test was used to compare qualitative variables between groups. Variables that were distributed in a normal way were analyzed by student $t$ test and paired $t$ test, besides Mann-Whitney and Wilcoxon tests were performed for variables that did not have normal distribution. The normal distribution of all studied data was checked with Kolmogorov-Smirnov test. Repeated measure ANOVA evaluated the differences between the groups during different periods of time. The two-tailed $P$ value $<0.05$ was considered significant.

\section{Results}

The study flowchart is shown in Figure 1. Ninety-three patients with chemotherapy-induced hepatotoxicity were registered in the study. Eighty percent of patients were enrolled with elevated alanine aminotransferase (ALT) and remaining patients for elevated aspartate aminotransferase (AST), since there was no increase in bilirubin level. Based on gender and age, the participants were allocated in two arms. Ninety patients were able to be evaluated and completed the study; 45 patients randomly assigned to the intervention group and 45 to the control group. One patient refused to take any dose of milk thistle and 2 patients in the control group had rising of enzymes during the study that they were excluded from study analysis. In milk thistle arm, Livergol with $7 \mathrm{mg} / \mathrm{kg}$ daily was prescribed. The drug was administrated as tablets of 70 and $140 \mathrm{mg}$. the calculated number of tablets per dose was rounded to the nearest half or whole tablet. Around 75\% of patients of milk thistle group and in the placebo group
$85 \%$ of patients had grade 2 toxicity and the remaining patients in each group who enrolled in the study had grade 3 toxicity. No patients with grade 4 toxicity registered into the study. Seven patients in the milk thistle group and 10 patients in the placebo group received one tablet (70 $\mathrm{mg}$ ) per day; 24 patients in the milk thistle group and 20 patients in the placebo group received one tablet $(140 \mathrm{mg}$ ) per day; 10 in milk thistle and 8 in placebo received 2 tablets $(70+140 \mathrm{mg})$ per day; and 4 in milk thistle and 7 in the placebo received 3 tablets $(140+140+70 \mathrm{mg})$ per day. In the control group placebo tablets similar to Livergol in shape and color, were prescribed daily.

Children were between 3-15 years old. The mean age was 6.73 years in the milk thistle group and in the control group it was 6.51 years. The total number of females and males in this study were 45 patients (Total: 90). No statistically significant difference there was between age and gender in the study. Demographic variables in terms of age $(P=0.079)$ and gender $(P=0.058)$ in both groups were similar (Table 1$)$.

At baseline, AST and ALT levels between the milk thistle group and placebo group did not show a statistical difference ( $P=0.8$ and $P=0.4$ respectively). At day 35 and day 70 of the study, in milk thistle arm ALT and AST mean serum levels were lower than the placebo group $(P<0.001$; Figure 2). Mean total and direct bilirubin levels did not show a significant difference between the milk thistle group and placebo group at baseline, days 35 and 70 .

Mean reductions in intra-individual AST, and ALT over 35 and 70 days from baseline were evaluated by using difference scores. There was no significant difference in mean reduction of AST and ALT from day 0 to day 35 in

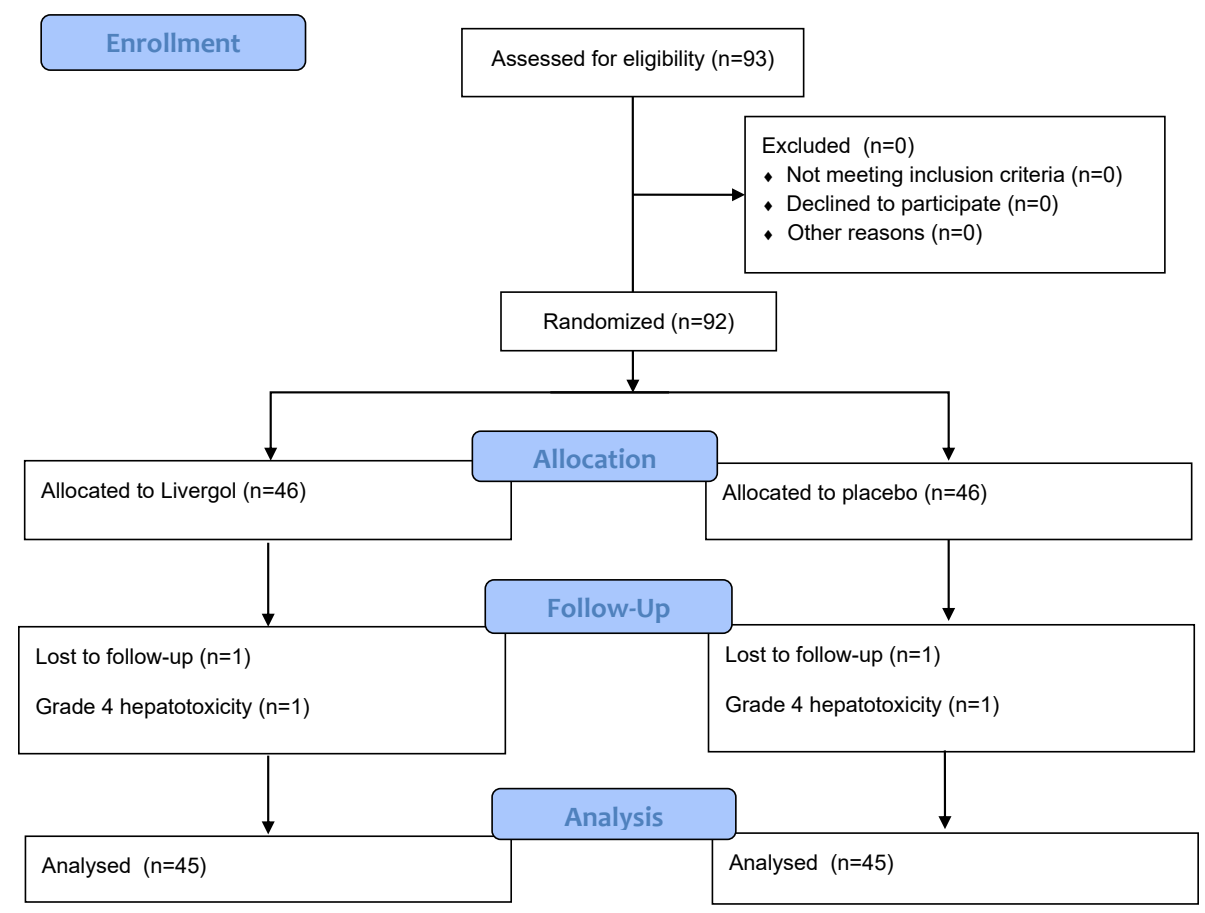

Figure 1. CONSORT flow diagram of the study. 
Table 1. Demographics by randomized group

\begin{tabular}{lcc}
\hline Variable & Livergol $(\mathbf{n}=\mathbf{4 5})$ & Placebo $(\mathbf{n}=\mathbf{4 5})$ \\
\hline Age $(\mathrm{y})$ & & \\
$3-5$ & $12(26.7 \%)$ & $12(26.7 \%)$ \\
$5-10$ & $27(60 \%)$ & $29(64.4 \%)$ \\
$>10$ & $6(13.3 \%)$ & $4(8.9 \%)$ \\
Mean/y & 6.73 & 6.51 \\
Gender & & \\
Male & 21 & 27 \\
Female & 24 & 18 \\
Risk group & & \\
Standard risk & 41 & 43 \\
High risk & 4 & 2 \\
Eligibility (elevated) & & 8 \\
AST (IU/L) & 10 & 37 \\
ALT (IU/L) & 35 & 0 \\
Total bilirubin (mg/dL) & 0 & \\
\hline
\end{tabular}

the placebo group. However, in the milk thistle arm mean reduction of AST and ALT from day 0 (before treatment) to day 35 ( mean reduction 38.68 and 91.40 , respectively with Sig. 2-tailed 0.000 ) and from day 0 to day 70 had significant reduction ( mean reduction 44.15 and 102.68 respectively with Sig. 2-tailed 0.000).

In contrast to other studies, complications such as diarrhea due to medication were not observed in this study. It should be noted that the weekly cumulative dose of chemotherapy drugs did not differ significantly in both groups. We did not have any increase in enzymes level in the milk thistle arm of study.

\section{Discussion}

Milk thistle effects on liver function and elevated liver enzymes were evaluated in our study. To our knowledge, the effects of milk thistle on liver function in patients with leukemia have been evaluated in few studies. For the first time we introduced Livergol as an antioxidant for treatment of hepatotoxicity. We found that short course of Livergol is effective in decreasing liver enzymes and with Livergol there is no need to chemotherapy doses reduction or delay in therapy courses.

Post-chemotherapy rising of liver enzymes is one of common side effects of chemotherapy and usually results in discontinuation of chemotherapy or dose reduction that both of them associate with poor outcome.

In the study performed by Ladas et al no significant changes in mean ALT, AST, or total bilirubin at day 28, however, at day 56 , the milk thistle group had a significantly lower AST and ALT (14). They concluded that in children with ALL and chemotherapy-induced hepatotoxicity, milk thistle was associated with a trend toward significant reductions in liver toxicity parameters and milk thistle did not antagonize the effects of chemotherapy agents used for the treatment of ALL (14). The Livergol protective effects may be due to the blocking of toxin receptors on hepatocytes and free radical scavenging features. Moreover, inflammatory mediators such as tumor necrosis

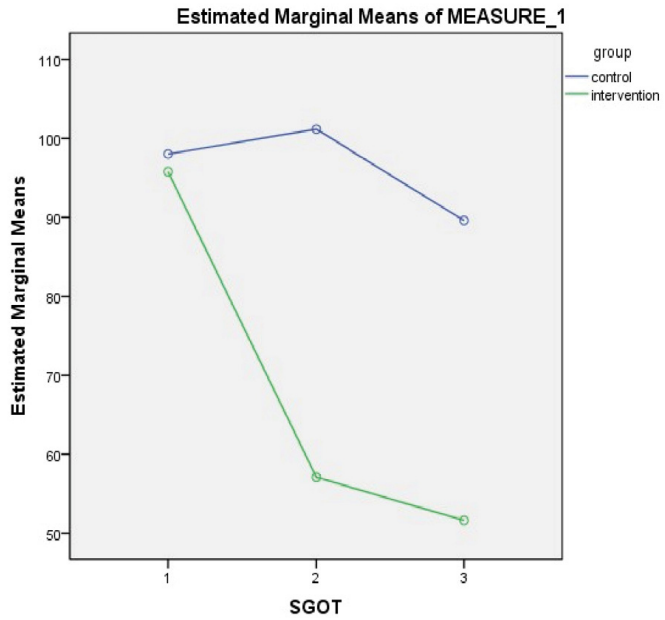

Figure 2. Mean AST and ALT level during 70 days in both groups.

factor, nitrous oxide, interleukin-6, and interleukin-1 receptor antagonist were regulated by milk thistle (24-26). There are more studies that used milk thistle for treatment of oxidative stresses in different diseases, but yet there is no consensus about the therapeutic benefits.

Moreover, Sule et al reported that milk thistle has been effective in some of the pediatric ALL patients to prevent and treat the chemotherapy-induced hepatotoxicity by reduction in AST and ALT serum levels as compared to control subjects (23). Based on our results there was no reduction in AST and ALT levels of patients in placebo group during 70 days (Figure 2). In the Livergol group the elimination rate of ALT and AST level between day 35 and 70 is much lower than the reduction at day 0 to 35 because patients who take Livergol just for $1^{\text {st }} 35$ days. This suggests that milk thistle is effective in improvement of liver function while the effect of Livergol can even continue after patients stopped taking it. Elevation of liver enzyme in $60 \%$ of our patients shows that we should monitor routinely liver function in all of ALL patients over maintenance phase of chemotherapy. As recent studies show, maintenance phase is as important as other intensive phases of ALL chemotherapy (27) and any dose reduction or discontinuation of chemotherapy increase the risk of relapse (28). One of the helpful medications for continuing and nonstop chemotherapy may be Livergol.

\section{Conclusion}

As our results showed the beneficial effects of milk thistle in chemotherapy-induced hepatotoxicity, which could be prescribed as a supportive treatment in addition to chemotherapy, leads to hepatotoxicity recovery in the short term. Future clinical trials should be conducted to explore longtime effect of Livergol in leukemia patients and to determine if there is any need for prophylactic administration of antioxidants.

\section{Study limitations}

This study was restricted to the maintenance phase, so sample size was relatively small. 


\section{Authors' contribution}

Principal investigators were FG and MN and PS. FG, PS and JR were included in preparing the concept and design. SH and JR revisited the manuscript and critically evaluated the intellectual contents. All authors participated in preparing the final draft of the manuscript, revised the manuscript and critically evaluated the intellectual contents. All authors have read and approved the content of the manuscript and confirmed the accuracy or integrity of any part of the work.

\section{Conflicts of interest}

The authors have indicated that they have no conflicts of interest regarding the contents of this article.

\section{Ethical considerations}

Ethical issues (including plagiarism, data fabrication, double publication) completely observed by the authors.

\section{Funding/Support}

None.

\section{References}

1. Stat Bite: Estimated New Leukemia Cases in 2008. J Natl Cancer Inst. 2008;100:531. doi:10.1093/jnci/djn111.

2. Tao YF, Pang L, Du XJ, Sun LC, Hu SY, Lu J, et al. Differential mRNA expression levels of human histone-modifying enzymes in normal karyotype B cell pediatric acute lymphoblastic leukemia. Int J Mol Sci. 2013;14:3376-3394. doi: 10.3390/ ijms14023376.

3. Ribera JM. Acute lymphoblastic leukemia. In: Hentrich M, Barta K, editors. HIV-Associated Hematological Malignancies. Springer: 2016. p. 145-151.doi: 10.1007/978-3-319-26857-6.

4. Hagag A, Abdel E, Aly R. Prognostic value of plasma levels of thrombomodulin and von Willebrand factor in Egyptian children with acute lymphoblastic leukemia. J Oncol Pharm Pract. 2014;20:356-61. doi: 10.1177/1078155213508439.

5. Mahoney DH Jr, Shuster JJ, Nitschke R, Lauer SJ, Steuber $\mathrm{CP}$, Winick $\mathrm{N}$, et al. Acute neurotoxicity in children with B-precursor acute lymphoid leukemia: an association with intermediate-dose intravenous methotrexate and intrathecal triple therapy. J Clin Oncol. 1998;16:1712-22. doi: 10.1200/ JCO.1998.16.5.1712.

6. Morris-Stiff G, White AD, Gomez D, Cameron IC, Farid S, Toogood GJ, et al. Nodular regenerative hyperplasia (NRH) complicating oxaliplatin chemotherapy in patients undergoing resection of colorectal liver metastases. Eur J Surg Oncol. 2014;40:1016-20. doi: 10.1016/j.ejso.2013.09.015.

7. Cheng KK. Association of plasma methotrexate, neutropenia, hepatic dysfunction, nausea/vomiting and oral mucositis in children with cancer. Eur J Cancer Care. 2008;17:306-11. doi: 10.1111/j.1365-2354.2007.00843.

8. Ebbesen MS, Nygaard U, Rosthøj S, Sørensen D, Nersting J, Vettenranta K, et al. Hepatotoxicity During Maintenance Therapy and Prognosis in Children With Acute Lymphoblastic Leukemia. J Pediatr Hematol Oncol. 2017;39:161-6. doi: 10.1097/MPH.0000000000000733.

9. Comelli MC, Mengs U, Schneider C, Prosdocimi M. Toward the definition of the mechanism of action of silymarin: activities related to cellular protection from toxic damage induced by chemotherapy. Integr Cancer Ther. 2007;6:120-9. doi: $10.1177 / 1534735407302349$.

10. Rambaldi A, Jacobs BP, Gluud C. Milk thistle for alcoholic and/ or hepatitis B or C virus liver diseases. Cochrane Database Syst Rev. 2007 Oct 17;(4):CD003620.

11. Invernizzi R, Bernuzzi S, Ciani D, Ascari E. Silymarine during maintenance therapy of acute promyelocytic leukemia. Haematologica. 1993;78:340-341.
12. Giovanni L, Carlo Castruccio C, Laura G, Ignazio B. Potential therapeutic effects of milk thistle. EMBJ. 2015;10:168-72. doi: 10.3269/1970-5492

13. Tamayo C, Diamond S. Review of clinical trials evaluating safety and efficacy of milk thistle. Integr Cancer Ther. 2007; 6:146-57. doi: 10.1177/1534735407301942.

14. Ladas EJ, Kroll DJ, Oberlies NH, Cheng B, Ndao DH, Rheingold $\mathrm{SR}$, et al. A randomized, controlled, double-blind, pilot study of milk thistle for the treatment of hepatotoxicity in childhood acute lymphoblastic leukemia. Cancer. 2010;116:506-13. doi: 10.1002/cncr.24723.

15. National Cancer Institute Common Toxicity Criteria. National Cancer Institute; 2009.

16. Piscitelli SC, Formentini E, Burstein AH, Alfaro R, Jagannatha $\mathrm{S}$, Falloon J. Effect of milk thistle on the pharmacokinetics of indinavir in healthy volunteers. Pharmacotherapy. 2002; 22:551-6. doi: 10.1592/phco.22.8.551.33205.

17. Zuber R, Modriansky M, Dvorak Z, Rohovsky P, Ulrichova J, Simanek $\mathrm{V}$, et al. Effect of silybin and its congeners on human liver microsomal cytochrome P450 activities. Phytother Res. 2002;16:632-8. doi: 10.1002/ptr.1000.

18. Pepping J. Milk thistle: Silybum marianum. Am J Health Syst Pharm. 1999;56:1195-7.doi: 10.1093/ajhp/56.12.1195.

19. Rainone F. Milk thistle. Am Fam Physician. 2005;72:12851292

20. Hagag AA, Elgamsy MA, El-Asy HM, Mabrouk MM. Protective Role of silymarin on hepatic and renal toxicity induced by MTX based chemotherapy in children with acute lymphoblastic leukemia. Mediterr J Hematol Infect Dis. 2016;8:e2016043. doi: 10.4084/MJHID.2016.043.

21. Bouhalit S, Kechrid Z. Protective effect of silymarin extracted from Silybum marianum seeds upon nickel-induced hepatotoxicity in albino Wistar rats. Ann Microbiol Immunol. 2018;1:1005.

22. McBride A, Augustin KM, Nobbe J, Westervelt P. Silybum marianum (milk thistle) in the management and prevention of hepatotoxicity in a patient undergoing reinduction therapy for acute myelogenous leukemia. J Oncol Pharm Pract. 2012; 18:360-5. doi: 10.1177/1078155212438252.

23. Sule U, Turan B, Betul T, Fatma G, Mualla C. The concomitant use of milk thistle in pediatric acute lymphoblastic leukemia for the prevention and treatment chemotherapy related hepatotoxicities. J Clin Oncol. 2015;33:15. doi: 10.1200/ jco.2015.33.

24. Manna SK, Mukhopadahyay A, Van NT, Aggarwal B. Silymarin suppresses TNF-induced activation of NF-kappa B, c-jun N-terminal kinase and apoptosis. J Immunol. 1999;163(12): 6800-9.

25. Kaur G, Athar M, Alam MS. Dietary supplementation of silymarin protects against chemically induced nephrotoxicity, inflammation and renal tumor promotion response. Invest New Drugs. 2010;28:703-13. doi: 10.1007/s10637-009-9289-6.

26. Toklu HZ, Tunali Akbay T, Velioglu-Ogunc A, Ercan F, Gedik $\mathrm{N}$, Keyer-Uysal M, et al. Silymarin, the antioxidant component of Silybum marianum, prevents sepsis-induced acute lung and brain injury. J Surg Res. 2008;145:214-22. doi: 10.1016/j. jss.2007.03.072

27. Schmiegelow K, Nielsen SN, Frandsen TL, Nersting J. Mercaptopurine/Methotrexate maintenance therapy of childhood acute lymphoblastic leukemia: clinical facts and fiction. J Pediatr Hematol Oncol. 2014;36:503-17. doi: 10.1097/MPH.0000000000000206.

28. Schmiegelow K, Forestier E, Kristinsson J, Söderhäll S, Vettenranta K, Weinshilboum $R$, et al. Thiopurine methyltransferase activity is related to the risk of relapse of childhood acute lymphoblastic leukemia. J Leukemia. 2009;3:557-64. doi: 10.1038/leu.2008.316 